\title{
Recent Advances in High-Throughput Automated Powder Dispensing Platforms for Pharmaceutical Applications
}

\author{
SUPPORTING INFORMATION
}

AUTHOR NAMES

Matthew N. Bahr *†, Mark A. Morris §, Noah P. Tu ף, Aakankschit Nandkeolyar †\#

\section{AUTHOR ADDRESSES}

† GlaxoSmithKline, Pharmaceutical Research and Development, 1250 South Collegeville Road, Collegeville, Pennsylvania 19426, United States

$\S$ Vertex Pharmaceuticals Inc, 50 Northern Avenue, Boston, Massachusetts 02210, United States

I AbbVie Inc, 1 North Waukegan Road, North Chicago, Illinois 60064, United States

\# Drexel University, 3141 Chestnut Street, Philadelphia, Pennsylvania 19104, United States 


\section{CHEMSPEED AUTODOSE / AUTODISPENSE FUNCTIONALITY.}

The GDU-S SWILE at GSK has an application file installed that utilizes an "AutoDose" logic, and operates by using a glass capillary tube and piston assembly, known as a SWIN. There are three different SWIN sizes available on this system: small (0.6 mm ID), medium (1.0 mm ID), and large (2.0 mm ID). The SWIN takes up powder in small chunks as the void in the capillary is plunged into a bed of powder in the source bottle, and then measured using an overhead balance installed within the GDU-S SWILE. The powder is released into the target vial where it is weighed by an on-deck precision analytical balance to verify if the target amount was met. If the logic is satisfied that the target is met, the unit proceeds to fill subsequent vials, otherwise it will continue dispensing powder with the SWIN until complete. The GDU-Pfd at GSK has an

application file that utilizes an "AutoDispense" logic, and uses a traditional hopper/auger design (called a container) that operates via a rapid open/close action of a crescent-shaped opening at the bottom of the container. This allows powder to fall from the container in controlled amounts while continuously evaluating the mass dispensed through a series of machine logic if/then steps until the target amount is reached.

\section{CHEMSPEED GDU-S SWILE PLATFORM OBSERVATIONS}

Through the course of the dispensing activity, if the software assessed that the dispense amounts were not being achieved after several attempts, a decision was made to attempt the dispense using a different size SWIN to achieve the desired result. In 33 cases, the software reverted to medium SWINs at the $0.5 \mathrm{mg}$ target when the desired amount was not being satisfied. Similarly, there were 28 cases where the medium SWIN was replaced with a large SWIN at the $3.0 \mathrm{mg}$ target amount in order to satisfy the dispense target. In all cases where the SWIN was replaced, the mass 
dispensed successfully with the replacement SWIN. The data shown in Figure S1 illustrates how the GDU-S SWILE performed for each individual dispense throughout the entire run. In general, the dispenses were well within expectations. The exceptions are typically seen with materials such as D-Mannitol and sodium chloride at the upper target amounts. The most difficult powder to dispense was sodium chloride. The presumption is that the large cubic crystal does not pack well inside the medium and large SWIN tips. When material is successfully packed into the larger tip, the systematic plunging of the inner piston has difficulty with controlling the loss of these large crystals in the stepwise fashion that the dispensing is conducted.

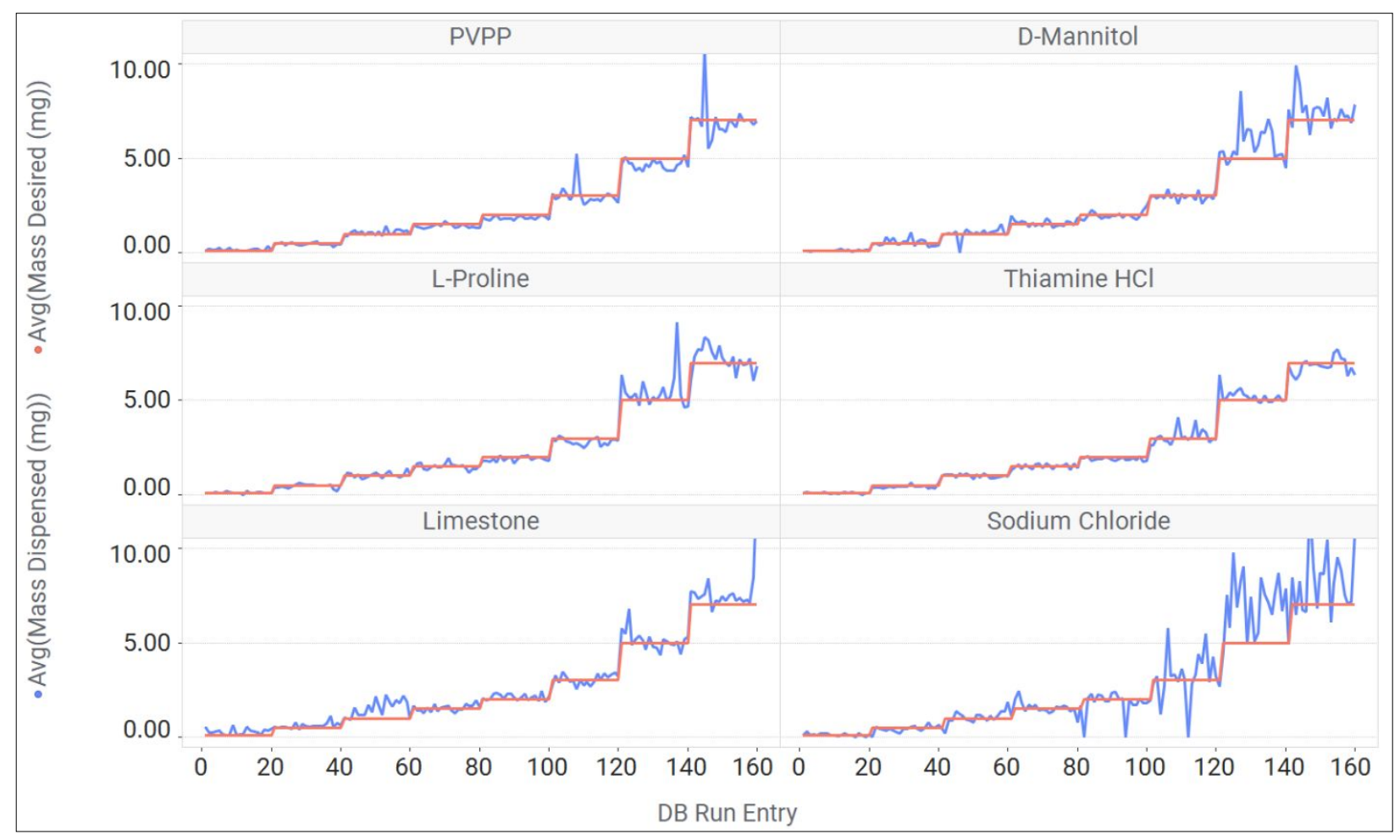

Figure S1. Line Chart of Chemspeed GDU-S SWILE Dispenses Performed at GSK. Red line shows mass desired in comparison to mass dispensed. 


\section{CHEMSPEED APPLICATION FILE DOSING PARAMETERS}

The traditional Chemspeed SWING software relies on the user to manually entering settings for rough dosing (RD) and fine dosing (FD) speed, acceleration, amplitude, and chunk size settings. For the AbbVie system, the following dispensing parameters (Table S1) were programmed into the Chemspeed SWING application file based on preliminary testing. For all targeted weights, FD amount was set at $10 \mathrm{mg}$, equilibration time was set at 4 seconds, and chunk size was set at 1 mg.

Table S1. Chemspeed SWING Dispense Parameters (AbbVie).

\begin{tabular}{|c|c|c|c|c|c|c|c|}
\hline Material & $\begin{array}{c}\text { Target } \\
\text { Amt } \\
\text { (mg) }\end{array}$ & $\begin{array}{c}\text { RD } \\
\text { Speed } \\
\text { (rpm) }\end{array}$ & $\begin{array}{c}\text { RD } \\
\text { Accel } \\
\left(\mathbf{s}^{-1}\right)\end{array}$ & $\begin{array}{l}\text { RD Ampl } \\
(\%)\end{array}$ & $\begin{array}{c}\text { FD } \\
\text { Speed } \\
\text { (rpm) }\end{array}$ & $\begin{array}{c}\text { FD Accel } \\
\left(\mathbf{s}^{-1}\right)\end{array}$ & $\begin{array}{c}\text { FD Ampl } \\
(\%)\end{array}$ \\
\hline \multirow{3}{*}{ PVPP } & 50 & 80 & 40 & 30 & 80 & 40 & 20 \\
\hline & 10 & 80 & 40 & 30 & 80 & 40 & 25 \\
\hline & 2 & 80 & 40 & 30 & 80 & 40 & 25 \\
\hline \multirow{3}{*}{ D-Mannitol } & 50 & 80 & 40 & 40 & 80 & 30 & 20 \\
\hline & 10 & 80 & 40 & 80 & 80 & 40 & 40 \\
\hline & 2 & 80 & 40 & 80 & 80 & 30 & 30 \\
\hline \multirow{3}{*}{ L-Proline } & 50 & 80 & 40 & 90 & 80 & 40 & 50 \\
\hline & 10 & 80 & 40 & 90 & 80 & 40 & 50 \\
\hline & 2 & 80 & 40 & 90 & 80 & 40 & 50 \\
\hline \multirow{3}{*}{$\begin{array}{c}\text { Thiamine } \\
\mathrm{HCl}\end{array}$} & 50 & 40 & 20 & 100 & 100 & 48 & 100 \\
\hline & 10 & 40 & 20 & 100 & 100 & 48 & 100 \\
\hline & 2 & 40 & 20 & 100 & 150 & 48 & 100 \\
\hline \multirow{3}{*}{ Limestone } & 50 & 40 & 20 & 100 & 80 & 20 & 100 \\
\hline & 10 & 40 & 20 & 100 & 150 & 48 & 100 \\
\hline & 2 & 40 & 20 & 100 & 150 & 48 & 100 \\
\hline \multirow{3}{*}{$\mathrm{NaCl}$} & 50 & 80 & 40 & 30 & 80 & 40 & 25 \\
\hline & 10 & 80 & 40 & 30 & 80 & 40 & 25 \\
\hline & 2 & 80 & 40 & 30 & 80 & 40 & 25 \\
\hline
\end{tabular}

\section{METTLER TOLEDO PLATFORM SETTING SELECTIONS}


The system deployed at Vertex underwent a detailed study investigating 1,080 dispenses with a tolerance setting of 5\% with the Advanced algorithm, and an additional 1,080 dispenses with a tolerance setting of $10 \%$ with the Standard algorithm. The 5\% Advanced algorithm consistently dispensed with higher precision at the $2 \mathrm{mg}$ target dose. However, at the $10 \mathrm{mg}$ and $50 \mathrm{mg}$ targets, there is no statistical significance in the precision.

The 5\% Advanced setting consistently under-dispenses for all materials and target doses, with the only exception being sodium chloride at the $10 \mathrm{mg}$ target. The $10 \%$ Standard setting dispenses, on average, were closer to the target amount for the $10 \mathrm{mg}$ and $50 \mathrm{mg}$ dispenses.

Table S2. Summary Averages and Standard Devaitions of Tolerance and Algorithm Settings.

\begin{tabular}{|c|c|c|c|c|c|c|c|}
\hline Material & $\begin{array}{c}\text { Target Dose } \\
\text { (mg) }\end{array}$ & $2 \mathrm{mg}$ & $2 \mathrm{mg}$ & $10 \mathrm{mg}$ & $10 \mathrm{mg}$ & $50 \mathrm{mg}$ & $50 \mathrm{mg}$ \\
\hline & $\begin{array}{l}\text { Tolerance } \\
\text { Algorithm }\end{array}$ & $\begin{array}{c}5 \% \\
\text { Adv } \\
\text { Algo }\end{array}$ & $\begin{array}{c}10 \% \\
\text { Std } \\
\text { Algo }\end{array}$ & $\begin{array}{c}5 \% \\
\text { Adv } \\
\text { Algo }\end{array}$ & $\begin{array}{c}10 \% \\
\text { Std } \\
\text { Algo }\end{array}$ & $\begin{array}{c}5 \% \\
\text { Adv } \\
\text { Algo }\end{array}$ & $\begin{array}{c}10 \% \\
\text { Std } \\
\text { Algo }\end{array}$ \\
\hline \multirow{2}{*}{$\begin{array}{c}\text { PVPP } \\
\text { (Kollidon) }\end{array}$} & $\begin{array}{c}\text { Actual Dose } \\
(\mathrm{mg})\end{array}$ & 1.93 & 2.01 & 9.57 & 9.89 & 48.31 & 49.94 \\
\hline & Std Dev & 0.0 & 0.1 & 0.0 & 0.3 & 0.3 & 0.2 \\
\hline \multirow{2}{*}{ D-mannitol } & $\begin{array}{c}\text { Actual Dose } \\
(\mathrm{mg})\end{array}$ & 1.95 & 2.07 & 9.92 & 9.91 & 48.38 & 49.85 \\
\hline & Std Dev & 0.1 & 0.1 & 0.5 & 0.3 & 0.6 & 0.4 \\
\hline \multirow[t]{2}{*}{ L-proline } & $\begin{array}{l}\text { Actual Dose } \\
(\mathrm{mg})\end{array}$ & 1.90 & 2.03 & 9.69 & 9.95 & 48.03 & 49.83 \\
\hline & Std Dev & 0.1 & 0.1 & 0.2 & 0.2 & 0.5 & 0.4 \\
\hline \multirow{2}{*}{$\begin{array}{c}\text { Thiamine } \\
\mathrm{HCl}\end{array}$} & $\begin{array}{c}\text { Actual Dose } \\
\text { (mg) }\end{array}$ & 1.93 & 2.03 & 9.59 & 10.00 & 48.51 & 49.92 \\
\hline & Std Dev & 0.0 & 0.1 & 0.0 & 0.1 & 0.3 & 0.2 \\
\hline \multirow[t]{2}{*}{ Limestone } & $\begin{array}{l}\text { Actual Dose } \\
(\mathrm{mg})\end{array}$ & 1.95 & 2.10 & 9.80 & 10.07 & 48.18 & 50.23 \\
\hline & Std Dev & 0.1 & 0.2 & 0.3 & 0.7 & 0.4 & 2.0 \\
\hline \multirow{2}{*}{$\mathrm{NaCl}$} & $\begin{array}{l}\text { Actual Dose } \\
\text { (mg) }\end{array}$ & 1.94 & 2.91 & 10.68 & 10.28 & 49.60 & 50.03 \\
\hline & Std Dev & 0.0 & 0.6 & 1.3 & 1.2 & 2.0 & 4.4 \\
\hline
\end{tabular}


The 2,160 dispenses conducted at these two tolerance and algorithm settings are presented in tabular form in Table S2 and visually in Figure S2. In this illustration, with the exception of sodium chloride, it is evident that both settings dispense accurately at all target three amounts. The $10 \%$ Standard algorithm dispenses were closer to the target amount, but with slightly larger variation for limestone and sodium chloride. The differences between the two settings were run through a one-way ANOVA (confidence interval of 5\%) and there was no statistical significance between the two settings overall.

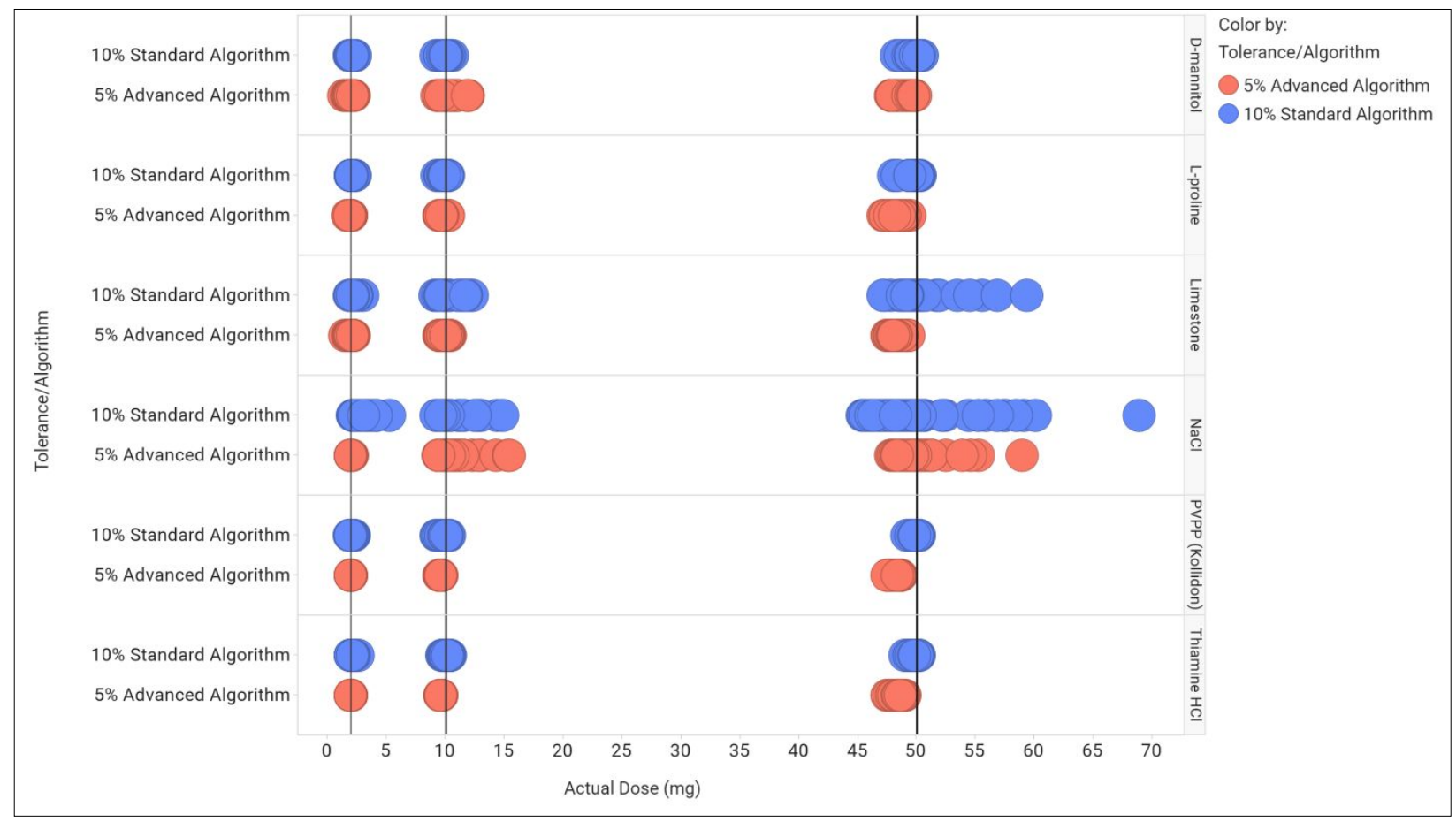

Figure S2. Actual Dispenses vs. Target Dose for Each Material, Comparing 5\% Advanced with 10\% Standard Algorithms. 


\section{BALANCE SPECIFICATIONS}

\section{METTLER TOLEDO CHRONECT:}

Measurement capacity: $0 \mathrm{~g}-220 \mathrm{~g}$

Resolution: $0.01 \mathrm{mg}$

\section{CHEMSPEED GDU-Pfd and GDU-S SWILE:}

Measurement capacity: $0 \mathrm{~g}-100 \mathrm{~g}$

Resolution: $0.1 \mathrm{mg}$

Linearity: $< \pm 0.2 \mathrm{mg}$

Calibration: External calibration weight of at least accuracy class E2

Dispensing Container Working Volume: $20 \mathrm{~mL}$

Lifetime: $>1,000$ dispenses of sodium carbonate, each requiring 50 extruder rotations.

The theoretical maximum dispensing accuracy and precision is given by the readibility of the balance, $\pm 0.1 \mathrm{mg}$ for the GDU-Pfd.

\section{ON-DECK EXTERNAL BALANCE WEIGHING MODULE:}

Measurement capacity, fine range: $0 \mathrm{~g}-111 \mathrm{~g}$

Resolution, fine range: $0.01 \mathrm{mg}$

Linearity: $< \pm 0.2 \mathrm{mg}$

\section{NOTE}

The authors offer the raw data powder dispensing file as a courtesy to the scientific community for research and modeling purposes. 